$\%$ A L. Ingber

$\% \mathrm{~T}$ Statistical mechanics of neocortical interactions. Derivation of short-term-memory capacity

$\%$ J Phys. Rev. A

$\%$ V 29

$\% \mathrm{P} 3346-3358$

$\% \mathrm{D} 1984$

\title{
Statistical mechanics of neocortical interactions. Derivation of short-term-memory capacity
}

\author{
Lester Ingber
}

Physical Studies Institute, Drawer W, Solana Beach, California 92075 and Institute for Pure and Applied Physical Sciences, University of California at San Diego, La Jolla, California 92093

(Received 7 November 1983; revised manuscript received 9 January 1984)

A theory developed by the author to describe macroscopic neocortical interactions demonstrates that empirical values of chemical and electrical parameters of synaptic interactions establish several minima of the path-integral Lagrangian as a function of excitatory and inhibitory columnar firings. The number of possible minima, their time scales of hysteresis and probable reverberations, and their nearestneighbor columnar interactions are all consistent with well-established empirical rules of human shortterm memory. Thus, aspects of conscious experience are derived from neuronal firing patterns, using modern methods of nonlinear nonequilibrium statistical mechanics to develop realistic explicit synaptic interactions.

PACS numbers 1983: 87.30.Gy, 87.10.+e, 02.50.+s, 05.50+q 


\section{INTRODUCTION}

One of the few well-established quantitative facts of neocortical information processing is that human short-term memory (STM), on the order of seconds, is limited to retention of $7 \pm 2$ items [1]. This is true even for apparently exceptional memory performers who, while they may be capable of more efficient encoding and retrieval of long-term memories (LTM), and while they may be more efficient in "chunking" larger patterns of information into single items, nevertheless also are limited to a capacity of $7 \pm 2$ items in their STM [2-4].

Previous papers have developed a theory of neocortical interactions [5-7], using modern methods of nonlinear nonequilibrium classical statistical mechanics $[8,9]$. The Appendix outlines the derivation of the key equations developed in these papers, but these papers should be consulted for extensive reference lists and more information on the biological, physical and mathematical issues.

This development in previous papers [6,7] has demonstrated that multiple hierarchies of several spatial-temporal scales of neocortical interactions can be consistently analyzed: Microscopic neuronal synaptic interactions, consistent with anatomical observations, are first spatially averaged over minicolumnar afferent and macrocolumnar efferent domains, defining a physiological "mesocolumn." These spatially ordered domains retain intimate contact with the original physical synaptic parameters, are consistent with observed columnar physiology, and are a suitable substrate for macroscopic spatialtemporal regions described by a path-integral Lagrangian formalism of coupled excitatory-inhibitory spatial-temporal firing states. Nearest-neighbor (NN) interactions among mesocolumns support regions of alternating columnar structures. Long-ranged influences from extrinsic and inter-regional afferents drive these short-ranged interactions, giving rise to columnar mechanisms affecting macroscopic activity.

Within neighborhoods of most-probable stationary firing minima determined by the Euler-Lagrange equations, the linearized field equations give rise to dispersion relations relating firing frequencies and spatial wave vectors [7]. Typical electroencephalographic (EEG) rhythms, wave numbers, and propagation velocities are nontrivially derived using only reasonable synaptic parameters. These mesoscopic relations are consistent with other studies which have derived similar macroscopic dispersion relations and have shown them to have strong empirical support [10]. A confluence of these sets of relations might represent an approximate linearized theory across several spatial scales which could be verifiable by existing EEG and magnetoencephalographic (MEG) measurements. The formation, stability, and interaction of spatial-temporal patterns of columnar firings can be explicitly calculated, to test hypothesized mechanisms relating to information processing. A detailed scenario has been calculated of columnar coding of external stimuli, short-term storage via hysteresis, and long-term storage via synaptic modification. This development supports the possibility of parallel processing of local information via microscopic circuits and of global patterned information via mesoscopic columnar mechanisms.

Here, these methods are further extended, and it is derived that, quite generally for realistic chemical and electrical synaptic parameters, the number of minima and the likely time scales of their interaction coincides with the observed limits of capacity of $7 \pm 2$. It is also calculated that the nearestneighbor columnar interactions support the sustenance of these columnar firing patterns of information. This is presented in Sec. II.

There have been previous suggestions that multiple roots of deterministic equations modeling neural nets might somehow be correlated with mechanisms of STM, but no specific functional forms or numerical estimates were made [11]. It is stressed in this theory [5,6] that there must be a consistent and detailed development of (a) synaptic interactions, (b) statistics of empirically observed columnar interactions, and (c) dynamics of the time evolution of this statistical nonlinear nonequilibrium system. The basic hypothesis is that memories-whether represented by specific microscopic circuitries or by relatively more global firing patterns - are encoded and secured in the environment of statistically favorable columnar firings, derived to be represented by a path-integral Lagrangian which establishes some important properties of STM capacity, duration, and interactions. There exists much support for the existence and independence of these kinds of globally parallel and selectively serial information processing $[3,4,12-14]$. 
These statistical mechanical techniques are quite general [9]. For example, they have been applied to study nucleon-nucleon velocity-dependent [15] Riemannian contributions to the binding energy of nuclear matter [16,17], to study the possibility of similar mechanisms in frustrated ferromagneticantiferromagnetic time-dependent interactions as encountered in spin-glasses [18], and to study the nonlinear nonequilibrium dynamics of financial markets [19].

\section{DERIVATION OF STM CAPACITY}

\section{A. STM stability}

For simplicity, initially consider the macroscopic prepoint-discretized Lagrangian $L$ derived in the Appendix, expressing the statistical evolution of mesocolumnar neuronal firings $M^{G}$ for excitatory $(G=E)$ and inhibitory $(G=I)$ type neurons.

$$
\begin{aligned}
& \underline{L}=\underline{L}^{E}+\underline{L}^{I}, \\
& \underline{L}^{G}=\left(\dot{M}^{G}-g^{G}\right)^{2} /\left(2 N g{ }^{G G}\right)+M^{G} J_{G} /(2 N \tau)-\underline{V}^{\prime}, \\
& g^{G}=-\tau^{-1}\left(M^{G}+N^{G} \tanh F^{G}\right), \\
& g^{G G}=\tau^{-1} N^{G} \operatorname{sech}^{2} F^{G}, \\
& F^{G}=\frac{\left(V^{G}-\sum_{G^{\prime}}\left(a_{G^{\prime}}^{G} v_{G^{\prime}}^{G} N^{G^{\prime}}-\frac{1}{2} A_{G^{\prime}}^{G} v_{G^{\prime}}^{G} M^{G^{\prime}}\right)\right)}{\left[\pi \sum_{G^{\prime}}\left[\left(v_{G^{\prime}}^{G}\right)^{2}+\left(\phi_{G^{\prime}}^{G}\right)^{2}\right]\left(a_{G^{\prime}}^{G} N^{G^{\prime}}+\frac{1}{2} A_{G^{\prime}}^{G} M^{G^{\prime}}\right)\right]^{1 / 2},} \\
& a_{G^{\prime}}^{G}=\frac{1}{2} A_{G^{\prime}}^{G}+B_{G^{\prime}}^{G},
\end{aligned}
$$

where $N^{G}$ is the number of neurons of type $G$ in an afferent minicolumn, $N=N^{E}+N^{I} \sim 110$, and $N^{*}=N^{* E}+N^{* I} \sim 10^{3} N$ is the number of neurons in an efferent macrocolumn. $\left\{V^{G}, v_{G^{\prime}}^{G}, \phi_{G^{\prime}}^{G}, A_{G^{\prime}}^{G}, B_{G^{\prime}}^{G}\right\}$ are mesocolumnar-averaged parameters derived from chemical and electrical synaptic parameters: $V^{G} \sim 10$ $\mathrm{mV}$ is the axonal threshold potential; $\left|v_{G^{\prime}}^{G}\right| \sim 0.1 \mathrm{mV}$ and $\phi_{G^{\prime}}^{G} 0.1 \mathrm{mV}$ are the mean and variance of potential distributed to the axonal trigger site of neuron $G$ from interaction with neuron $G^{\prime}$; $A_{G^{\prime}}^{G}=A_{G^{\prime}}^{* G} N^{*} / N \sim 10^{-2}-10^{-3} N^{*} / N$ and $B_{G^{\prime}}^{G}=B_{G^{\prime}}^{* G} N^{*} / N \sim \frac{1}{5} A_{G^{\prime}}^{G} N^{*} / N$ are the conductivity and spontaneous background across the synaptic cleft between neurons $G$ and $G^{\prime}$, consistently scaled to reflect the scaling of $M^{* G} \rightarrow M^{G}$ (see Appendix). Note that the Einstein convention of summing over repeated indices is not yet invoked in this section.

An examination of this derivation makes it clear that $M^{G}=M^{*}{ }^{G} N / N^{*}$ represents an efferent mesocolumnar firing. $M^{G}=N^{G}$ means all neurons in a mesocolumn are firing, $M^{G}=-N^{G}$ means they all are not firing. $\theta \dot{M}^{G}(t+\theta)=M^{G}(t+\theta)-M^{G}(t), \theta \leq \tau$, represents the change in the firing state of the afferent mesocolumn, thereby also representing a change in efferent firings at $t+\theta . V^{\prime G} \propto\left(\rho \nabla M^{G}\right)^{2}$ are NN interactions. $\tau \approx 10 \mathrm{msec}$ and $\rho \approx 10^{2} \mu \mathrm{m}$ are the temporal and spatial extents of mesocolumns. $J_{G}$ are constraints from intrinsic and extrinsic long-range fibers.

Three cases of neuronal firings are considered for further study. Since STM duration is still long relative to $\tau$, stationary solutions of $\underline{\underline{L}}$ will be investigated to determine how many stable minima $\ll \bar{M}^{G} \gg$ may simultaneously exist within this duration. Also, primarily individual mesocolumns will be studied. I.e., take the uniform limit of $\dot{\bar{M}}^{G}=0=\nabla \bar{M}^{G}$. Although the $\dot{\bar{M}}^{G}=0$ limit should only be taken for the midpoint-discretized Lagrangian $\underline{L}_{F}$, this is a small difference here, as will be demonstrated subsequently.

A model of dominant inhibition describes how minicolumnar firings are suppressed by their neighboring minicolumns. For example, this could be effected by developing NN mesocolumnar 
interactions [7], but here the averaged effect is established by inhibitory mesocolumns (IC) by setting $A_{E}^{I}=A_{I}^{E}=2 A_{E}^{E}=0.01 N^{*} / N$. Since there appears to be relatively little $I-I$ connectivity, set $A_{I}^{I}=0.0001 N^{*} / N$. The background synaptic noise is taken to be $B_{I}^{E}=B_{E}^{I}=2 B_{E}^{E}=10 B_{I}^{I}=0.002 N^{*} / N$. As minicolumns are observed to have $\sim 110$ neurons (visual cortex appears to have approximately twice this density) [20], and as there appear to be a predominance of $E$ over $I$ neurons [10], here take $N^{E}=80$ and $N^{I}=30$. Use $N^{*} / N=10^{3}, J_{G}=0$, and $V^{G}, v_{G^{\prime}}^{G}$, and $\phi_{G^{\prime}}^{G}$ as estimated previously. The "threshold factors" $F_{\mathrm{IC}}^{G}$ for this IC model are then

$$
\begin{aligned}
& F_{\mathrm{IC}}^{E}=\frac{\left(0.5 \bar{M}^{I}-0.25 \bar{M}^{E}+3.0\right)}{\pi^{1 / 2}\left(0.1 \bar{M}^{I}+0.05 \bar{M}^{E}+9.80\right)^{1 / 2}}, \\
& F_{\mathrm{IC}}^{I}=\frac{\left(0.005 \bar{M}^{I}-0.5 \bar{M}^{E}-45.8\right)}{\pi^{1 / 2}\left(0.001 \bar{M}^{I}+0.1 \bar{M}^{E}+11.2\right)^{1 / 2}} .
\end{aligned}
$$

In the prepoint-discretized deterministic limit, the threshold factors determine when and how smoothly the step-function forms $\tanh F_{\mathrm{IC}}^{G}$ in $g^{G}(t)$ in Eq. (2.1) change $M^{G}(t)$ to $M^{G}(t+\theta)$. $F_{\text {IC }}^{I}$ will cause afferent $\bar{M}^{I}$ to fire for most of its values, as $\bar{M}^{I} \sim-N^{I} \tanh F_{\text {IC }}^{I}$ will be positive for most values of $\bar{M}^{G}$ in $F_{\text {IC }}^{I}$, which is already weighted heavily with a term -45.8. Looking at $F_{\mathrm{IC}}^{E}$, it is seen that the relatively high positive values of efferent $\bar{M}^{I}$ require at least moderate values of positive efferent $\bar{M}^{E}$ to cause firings of afferent $\bar{M}^{E}$.

\section{-- Figure 1 --}

Figure 1 gives contour plots of $\bar{L}_{\mathrm{IC}}$ over $\bar{M}^{G}$ space, but cut off at various values to permit examination of various scales of resolution. The calculations presented here support the contention that neocortex functions at multiple hierarchies: While specific LTM information most likely is coded at the microscopic neuronal level, the mesoscopic scale most likely provides the context for multiple mostprobable firing patterns which process STM and which facilitates plastic synaptic encoding of LTM [7]. For example, the scale in Figure 1(a) is the smallest scale above which 2 stable regions of $\bar{L}_{\text {IC }}$ can be discerned. Higher cutoffs only would portray contours running across $\bar{M}^{G}$ space or minima whose valleys lie above these cutoffs. Previous papers have presented plots of similar Lagrangians at larger cutoffs and without cutoffs. E.g., $\tau \bar{L}$ can range from 0 to values $>10^{3}[6,7]$. As will be discussed subsequently in Sec. II.2, realistic constraints on STM duration dictate that only values of $\tau \bar{L} \leq 0.04$ are of interest here. Figure 1(b) is the largest scale at which one minimum can be discerned for all lower cutoffs. Figure 1(c) confirms this at a smaller scale.

Detailed calculations demonstrate that $\underline{L}^{E}$ or $\underline{L}^{I}$ separately typically give rise to more multiple minima, $\approx 10$, than permitted by their sum $\bar{L}$ at this resolution. This "loss" of minima apparently is an interesting consequence of $E-I$ competition at the mesoscopic scale. For example, since $\bar{L}^{G}$ scales as $N^{G} / N$ for relatively large $\bar{M}^{G}, \underline{L}^{E}$ dominates due to the larger $\bar{M}^{E}$ in its means $g^{E}$. For relatively small $\bar{M}^{G}, g^{G}$ typically is small if there are several multiple minima in $\bar{L}^{G}$, since most of the minima are found to cluster about the origin. Therefore, $\bar{L}^{G}$ scales as $\left(N^{G}\right)^{-1}$ from the variances $\left(g^{G G}\right)^{-1}$, and $\underline{L}^{I}$ dominates for small $\bar{M}^{G}$ due to the larger fluctuations of $\bar{L}^{I}$.

It is discovered that more minima of $\bar{L}$ are created, or "restored," if the numerator of $F^{G}$ in Eq. (2.1) contains terms only in $\bar{M}^{G}$, tending to center $\bar{L}$ about $\bar{M}^{G}=0$. Of course, any mechanism producing more as well as deeper minima is statistically favored. However, this particular "centering" mechanism has plausible support: $M^{G}(t+\tau)=0$ is the state of afferent firing with highest statistical weight. I.e., there are more combinations of neuronal firings, $\sigma_{j}= \pm 1$, yielding this state than any other $M^{G}(t+\tau)$, e.g., $\sim 2^{N^{G}+1 / 2}\left(\pi N^{G}\right)^{-1 / 2}$ relative to the states $M^{G}= \pm N^{G}$. Similarly, $M^{* G}(t)$ is the state of efferent firing with highest statistical weight. Therefore, it is natural to explore mechanisms which favor common highly weighted efferent and afferent firings in ranges consistent with favorable firing threshold 
factors $F^{G} \approx 0$. Another effect of this centering mechanism apparently is to shift minima of $\underline{L}^{G}$ closer together, permitting them to often cooperate instead of competing.

This centering effect of the IC model, labeled here as the $\mathrm{IC}^{\prime}$ model, is quite easy for neocortex to accommodate. For example, this can be accomplished simply by readjusting the synaptic background noise from $B_{E}^{G}$ to $B_{E}^{\prime}$,

$$
B_{E}^{\prime G}=\frac{\left[V^{G}-\left(\frac{1}{2} A_{I}^{G}+B_{I}^{G}\right) v_{I}^{G} N^{I}-\frac{1}{2} A_{E}^{G} v_{E}^{G} N^{E}\right]}{v_{E}^{G} N^{G}}
$$

for both $G=E$ and $G=I$. In general, $B_{E}^{G}$ and $B_{I}^{G}$ (and possibly $A_{E}^{G}$ and $A_{I}^{G}$ due to actions of neuromodulators, and $J_{G}$ constraints from long-ranged fibers) are available to zero the constant in the numerator, giving an extra degree(s) of freedom to this mechanism. (If $B_{E}^{\prime G}$ would be negative, this leads to unphysical results in the square-root denominator of $F^{G}$. Here, in all examples where this occurs, it is possible to instead find positive ${B^{\prime}}_{I}^{G}$ to appropriately shift the numerator of $F^{G}$.) In this context, it is empirically observed that the synaptic sensitivity of neurons engaged in selective attention is altered, presumably by the influence of chemical neuromodulators on postsynaptic neurons [21].

By this centering mechanism, $B_{E}^{\prime E}=1.38$ and ${B^{\prime}}_{I}^{I}=15.3$, and $F_{\mathrm{IC}}^{G}$ is transformed to $F_{\mathrm{IC}}^{G}$,

$$
\begin{aligned}
& F_{\mathrm{IC}}^{E}=\frac{\left(0.5 \bar{M}^{I}-0.25 \bar{M}^{E}\right)}{\pi^{1 / 2}\left(0.1 \bar{M}^{I}+0.05 \bar{M}^{E}+10.4\right)^{1 / 2}}, \\
& F_{\mathrm{IC}}^{I}=\frac{\left(0.005 \bar{M}^{I}-0.5 \bar{M}^{E}\right)}{\pi^{1 / 2}\left(0.001 \bar{M}^{I}+0.1 \bar{M}^{E}+20.4\right)^{1 / 2}} .
\end{aligned}
$$

Note that, aside from the enforced vanishing of the constant terms in the numerators of $F_{\mathrm{IC}^{\prime}}^{G}$, the only other changes in $F_{\mathrm{IC}}^{G}$ in Eq. (2.4) relative to $F_{\mathrm{IC}}^{G}$ in Eq. (2.2) is to moderately affect the constant terms in the denominators. Figure 1(d) illustrates the increase of minima of $\tau \bar{L}_{\mathrm{IC}}$ to 4 . The 2 minima clustered close to the origin are no longer discernible for $\tau \overline{\underline{I C}}_{\mathrm{IC}^{\prime}}>0.03$.

-- Figure 2 --

The other "extreme" of normal neocortical firings is a model of dominant excitation, effected by establishing excitatory mesocolumns (EC) by using the same parameters $\left\{B_{G^{\prime}}^{G}, v_{G^{\prime}}^{G}, \phi_{G^{\prime}}^{G}, A_{I}^{I}\right\}$ as in the IC model, but setting $A_{E}^{E}=2 A_{E}^{I}=2 A_{I}^{E}=0.01 N^{*} / N$. This yields

$$
\begin{aligned}
& F_{\mathrm{EC}}^{E}=\frac{\left(0.25 \bar{M}^{I}-0.5 \bar{M}^{E}-24.5\right)}{\pi^{1 / 2}\left(0.05 \bar{M}^{I}+0.10 \bar{M}^{E}+12.3\right)^{1 / 2}}, \\
& F_{\mathrm{EC}}^{I}=\frac{\left(0.005 \bar{M}^{I}-0.25 \bar{M}^{E}-25.8\right)}{\pi^{1 / 2}\left(0.001 \bar{M}^{I}+0.05 \bar{M}^{E}+7.24\right)^{1 / 2}} .
\end{aligned}
$$

The negative constant in the numerator of $F_{\mathrm{EC}}^{I}$ inhibits afferent $\bar{M}^{I}$ firings. Although there is also a negative constant in the numerator of $F_{\mathrm{EC}}^{E}$, the increased coefficient of $\bar{M}^{E}$ (relative to its corresponding value in $F_{\text {IC }}^{E}$ ), and the fact that $\bar{M}^{E}$ can range up to $N^{E}=80$, readily permits excitatory firings throughout most of the range of $\bar{M}^{E}$. This is illustrated in Figure 2(a), where 3 minima are possible.

Applying the centering mechanism to EC, $B_{I}^{\prime E}=10.2$ and $B_{I}^{\prime I}=8.62$. The net effect in $F_{\mathrm{EC}}^{G}$, in addition to removing the constant terms in the numerators of $F_{\mathrm{EC}}^{G}$, is to change the constant terms in the denominators: 12.3 in $F_{\mathrm{EC}}^{E}$ is changed to 17.2 in $F_{\mathrm{EC}}^{E}$, and 7.24 in $F_{\mathrm{EC}}^{I}$ is changed to 12.4 in $F_{\mathrm{EC}}^{I}$. Figure 2(b) illustrates that now 6 prominent minima are possible along a line through $\bar{M}^{G}=0$, and 2 others are at 
$\bar{M}^{G}= \pm N^{G}$. Each pair of minima above and below the $\bar{M}^{I}=0$ axis merge into single minima for $\tau \overline{\underline{L}}_{\mathrm{EC}^{\prime}}>0.02$, and these lose resolution for $\tau \overline{\underline{L}}_{\mathrm{EC}^{\prime}}>0.03$.

-- Figure 3 --

Now it is natural to examine a balanced case intermediate between IC and EC, labeled BC. This is accomplished by changing $A_{E}^{E}=A_{E}^{I}=A_{I}^{E}=0.005 N^{*} / N$. This yields

$$
\begin{aligned}
& F_{\mathrm{BC}}^{E}=\frac{\left(0.25 \bar{M}^{I}-0.25 \bar{M}^{E}-4.50\right)}{\pi^{1 / 2}\left(0.050 \bar{M}^{E}+0.050 \bar{M}^{I}+8.30\right)^{1 / 2}}, \\
& F_{\mathrm{BC}}^{I}=\frac{\left(0.005 \bar{M}^{I}-0.25 \bar{M}^{E}-25.8\right)}{\pi^{1 / 2}\left(0.001 \bar{M}^{I}+0.050 \bar{M}^{E}+7.24\right)^{1 / 2}} .
\end{aligned}
$$

Figure 3(a) illustrates that 3 minima are possible.

Applying the centering mechanism to $\mathrm{BC}, B_{E}^{\prime E}=0.438$ and $B_{I}^{\prime I}=8.62$. The net effect in $F_{\mathrm{BC}}^{G}$, in addition to removing the constant terms in the numerators of $F_{\mathrm{BC}}^{G}$, is to change the constant terms in the denominators: 8.30 in $F_{\mathrm{BC}}^{E}$ is changed to 7.40 in $F_{\mathrm{BC}}^{E}$, and 7.24 in $F_{\mathrm{BC}}^{I}$ is changed to 12.4 in $F_{\mathrm{BC}}^{I}$. Figure 3(b) illustrates that now 10 minima are possible! The 9 minima along the diagonal line lose resolution for $\tau \overline{\underline{B}}_{\mathrm{BC}^{\prime}}>0.01$ above $\bar{M}^{I}=0$ and for $\tau \bar{L}_{\mathrm{BC}^{\prime}}>0.02$ below $\bar{M}^{I}=0$.

\section{-- Figure 4 --}

The effects of considering the full Feynman Lagrangian $\bar{L}_{F}$, including all the Riemannian and other nonlinear corrections given in the Appendix, is illustrated in Figure 4(a) for BC'. The net effect is to slightly raise the threshold at which minima dissipate, to about $\tau \bar{L}_{\mathrm{BC}^{\prime}} \geq 0.03$, which is relevant for the duration of STM, discussed subsequently. However, note that the minima structure is essentially the same.

To demonstrate that multiple minima are not an effect of nonlinearities induced by the denominators of $F^{G}$, Figure 4(b) illustrates the net effect in $\bar{L}_{\mathrm{BC}}$ by dropping the $\bar{M}^{G}$ terms in the denominators of $F_{\mathrm{BC}^{\prime}}^{G}$. The valleys of minima are only slightly increased, i.e., they are not as deep relative to those in Figure 3(b). However, these denominators are still important contributions derived from synaptic interactions. E.g., even with the $\bar{M}^{G}$ terms dropped, the denominators contribute factors of $\sim \frac{1}{5}$ to $F_{\mathrm{BC}^{\prime}}^{G}$.

-- Figure 5 --

If $N^{*}$ is scaled larger or smaller, this effectively scales $A_{G^{\prime}}^{G}=A_{G^{\prime}}^{* G} N^{*} / N$ and $B_{G^{\prime}}^{G}=B_{G^{\prime}}^{* G} N^{*} / N$, disturbing the relatively sensitive balance that permits a few percent of efferent firings to affect their afferents. Then, the number of possible minima is typically reduced to one or two. If $N$ is scaled larger or smaller, the number of minima is altered and the duration of STM is affected, as discussed subsequently. However, for $N$ still in the range of a few hundred, the number of possible minima is not severely reduced. Figure 5 illustrates the case $N=220$, e.g., visual cortex: For model $\mathrm{BC}^{\prime}$, the number of prominent minima found is 11 . Note that the larger $N$ sharpens the minima and therefore the resolution of visual information processing.

These results are unchanged qualitatively for modest changes of any neocortical parameters. However, it is reasonable to conjecture that more drastic abnormal changes in the neocortical parameters might severely reduce the number of minima. This conjecture is based on calculations wherein $F^{G}$ do not possess the relatively sensitive balances allowing a few percent of efferent neurons to control firings in their afferents. In calculations using these unrealistic or abnormal parameters only one or two minima survive. 


\section{B. STM duration}

A hysteresis study affords a measure of the time scales on which these minima may be simultaneously present, e.g., before LTM mechanisms plastically change the columnar-averaged synaptic parameters $Z=\left\{A_{G^{\prime}}^{G}, B_{G^{\prime}}^{G}, v_{G^{\prime}}^{G}, \phi_{G^{\prime}}^{G}, V^{G}\right\}$, or before other firing patterns become prominent due to external stimulation $J_{G}[7]$.

For hysteresis to be prominent, the typical period within which synaptic parameters $Z$ are altered by $\Delta Z$ should be much greater than the relaxation period of $M^{G}$, but much less than the decay period for the system to jump or fluctuate between competing minima $\ll \bar{M}^{G} \gg$.

Time scales on which jumps between competing minima take place can be estimated by calculating the time of first passage between competing minima of $\underline{L}_{F}$, given by $\int d t \int d M P[M, t \mid \ll \bar{M} \gg, 0]$. An estimate of a stationary solution $P_{\text {stat }}$ to the Fokker-Planck differential equation (see Appendix) for the probability distribution $P$ of $M^{G}$ firings for an uncoupled mesocolumn, i.e., $V^{\prime}=0$, is given by the stationary limit of the short-time propagator,

$$
P_{\text {stat }} \approx N_{\text {stat }} g^{1 / 2} \exp (-C N \tau \underline{\underline{L}}),
$$

where $N_{\text {stat }}$ and $C$ are constant factors. This form is suggested because the minima of relevant examples, e.g., BC', essentially lie along a line in $\bar{M}^{G}$ space, effectively reducing the relevant calculation to a onedimensional Fokker-Planck equation [22]. An estimate of the approximation made in Eq. (2.7) is made by seeking values of constants $C$, such that the stationary Fokker-Planck equation is satisfied exactly. For model $\mathrm{BC}^{\prime}$, detailed investigation of contour plots of $C$ versus $\bar{M}^{G}$ demonstrates that there exists real positive $C$ which may only range from $\sim 10^{-1}$ to $\sim 1$, for which there exists unbroken contours of $C$ which pass through or at least border the line of minima. [At each point $\bar{M}^{G}$, Eq. (2.7) leaves a quadratic equation for $C$ to be solved.]

Thus, Eq. (2.7) defines a solution with potential $N^{2} \bar{L}=\int A d M$, drift $A$, and diffusion $N / \tau$. Stability, defined for $\delta M^{G}$ about a stationary state by $\delta \dot{M}^{G} \approx-A_{{ }_{G}} \delta M^{G}=-N^{2} \bar{L}_{, G G} \delta M^{G}$, is therefore equivalent to $\ll \bar{M} \gg$ being minima of $\underline{\underline{L}}$ as required for Sec. IIA.

An interesting interpretation may be given to those trajectories along $C<1$ : Take $C^{-1}$ to be close or equal to some integer. If several mesocolumns are considered to collectively carry a spatial pattern of information, this may be described approximately by $P_{\text {stat }}^{\backslash(d g}=\prod^{C^{-1}} P_{\text {stat }} \propto \exp \left(-C N \tau \Sigma^{C^{-1}} \underline{\underline{L}}\right) \approx$ $\exp (-N \tau \bar{L})$. Then $P_{\text {stat }}^{\backslash(d g}$, describing several mesocolumns within the reduced range $\left|\bar{M}^{\backslash(d g G}\right|=\left|\bar{M}^{G} / C\right| \leq N^{G}$, approximately satisfies the same Fokker-Planck equation along the line of its minima as does $P_{\text {stat }}$ describing a single mesocolumn along a solution trajectory with $C<1$. Therefore, there are conditions under which it is equally probable that STM may be processed by several mesocolumns as by a single mesocolumn. In the following it is assumed that $C=1$.

The time for first passage, $t_{v p}$, is estimated in analogy to a one-dimensional system as [22]

$$
\begin{aligned}
t_{v p} \approx \pi N^{-2}\left(\left|\underline{L}_{G G^{\prime}}\left(\ll \bar{M} \gg_{p}\right)\right| \underline{L}_{G_{G G^{\prime}}}\left(\ll \bar{M} \gg_{v}\right)\right)^{-1 / 2} \\
\quad \times \exp \left\{C N \tau\left[\underline{\underline{L}}\left(\ll \bar{M} \gg_{p}\right)-\bar{L}\left(\ll \bar{M} \gg_{v}\right)\right]\right\},
\end{aligned}
$$

where $\left\langle\bar{M} \gg_{v}\right.$ is the minimum at the valley of $\bar{L}$ in question, and $\ll \bar{M} \gg_{p}$ is the maximum at a peak separating two minima. Eqs. (2.7) and (2.8) are reasonable but crude estimates, and future numerical work must be done to detail the extent of their validity.

The exponential factor in Eq (2.8) can be quite large in some instances, and quite small in others. As noted previously [7], differences in $\bar{L}$ from valleys to peaks are still large relative to the Riemannian correction terms and relative to differential spatial-temporal contributions, thereby permitting this simpler analysis. However, values of $\tau \overline{\underline{L}}$ at maxima separating the far minima may be $>1$, thereby yielding a very large $t_{v p}$, typical of many physical systems undergoing hysteresis [7]. Relaxation times $t_{r}$ about this stationary state are estimated by $\left|g_{, G}^{G}\right|^{-1}[22]$, and are on the order of $\tau$. 
For changes $\Delta Z$ in synaptic parameters $Z=\left\{A_{j k}^{*}, B_{j k}^{*}, V_{j}, v_{j k}, \phi_{j k}, N^{*} G\right\}$ that transpire within a $\Delta t$ of several tenths of a second to seconds, e.g., during typical attention spans, hysteresis is more probable than simple jumps between minima if the following inequalities are satisfied. These estimates necessarily require more details of the system in addition to $t_{r}$ and $t_{v p}$ [22].

$$
\left|\frac{t_{r} \Delta t N^{2} \Delta \bar{L}_{, G}}{\Delta Z}\right|^{-1} \frac{N}{2} \gg \frac{\Delta Z}{\Delta t} \gg\left|\frac{N \tau t_{v p} \Delta \bar{L}}{\Delta Z}\right|^{-1} .
$$

For $\Delta Z$ approximately corresponding to a significant increase in the synaptic efficacy of one neuron per minicolumn, this typically leads to

$$
\frac{\Delta Z}{t_{r}} \gg \frac{\Delta Z}{\Delta t} \gg \frac{\Delta Z}{t_{v p}}
$$

where the last inequality may or may not hold, depending on the value of $t_{v p}$ used in Eq. (2.9).

Therefore, it is possible for hysteresis to be highly more probable than simple jump behavior to another firing state. This provides a mechanism whereby an extended temporal firing patterns of information can be processed beyond the time scale of relaxation periods, e.g., reverberation among several local minima. It is to be expected that the effects of $J_{G}(r ; t)$ on $\Delta Z(r ; t)$ create more complex examples of spatial-temporal hysteresis. These sustaining mechanisms may serve to permit other biochemical processes to store information for longer time periods as stable synaptic modifications, e.g., LTM. As detailed previously [7], changes in synaptic parameters $\Delta Z$ may duplicate the effects of $J_{G}$, providing a mechanism whereby columnar firings encode long-range firing constraints. If this encoding of firing patterns can establish itself on short enough time scales, then columnar coding of long-range firings could be a precursor mechanism initiating the centering mechanism above, especially across large regions of neocortex. Then, there would be a more uniform gradation of mechanism(s) establishing STM and LTM.

However, to address the issue of limited capacity of STM, it is reasonable to require that within time spans of tenths of a second to tens of seconds, simple jumps among minima are more probable than hysteresis. This permits all minima to be readily accessible during STM duration, in any ordering [2], at least more so than if hysteresis were more probable. In agreement with this empirical requirement, as detailed in the previous contour plots, it is found that $\tau\left[\underline{L}\left(\left\langle\bar{M} \gg_{p}\right)-\bar{L}\left(\left\langle\bar{M} \gg_{v}\right)\right] \sim 0.01-0.03\right.\right.$ for these models using empirical values for synaptic parameters. Then for $\left|\tau \bar{L}_{, G G^{\prime}}\right| \sim 10^{-3}, t_{v p} \sim 10 \tau-100 \tau$, on the order of several tenths of a second to a second. Use of the full Feynman Lagrangian $\bar{L}_{F}$ increases $t_{v p}$ slightly. For these relatively short $t_{v p}$ the second inequality in Eq. (2.10) is violated, and simple jumps are more probable than hysteresis, as required for STM.

Under conditions of serial processing [23], the deeper valleys of $\bar{L}$ representing the more likely firing states will be occupied first. In all cases considered here, several valleys are less likely than the others. This implies that the last several items in STM should be harder to encode (learn) and retain, with the possible exception of the last one or two items which represent the most recent shifting of firing patterns $\bar{M}^{G}$ to these minima $\ll \bar{M} \gg_{v}$ of $\bar{L}$. These conclusions are consistent with empirical observations [23], and are obtained independent of any other rehearsal mechanisms which may exist.

\section{-- Table I --}

Calculations in these models establish that the prefactor in Eq. (2.8) most often is $\sim \tau$. Table I demonstrates this by virtue of the fact that the determinant of the Hessian for points lying close to the diagonal in Figure $3(\mathrm{~b})$ is a very smooth function. However, note that points close to the corners $\bar{M}^{G}= \pm\left(N^{E}, N^{I}\right)$ have much more rapid variations. Therefore, minima at these corners, even when $\tau \underline{\underline{L}}\left(\ll \bar{M} \gg_{p}\right) \sim 0.01-0.03$, because of their sharp peaks, typically have $t_{v p}$ on the order of tens of seconds to jump to minima clustered on the diagonal. This is within the range where hysteresis is more probable for these minima. Therefore, minima at the corners of $\bar{M}^{G}$ space most likely do not contribute to STM, bringing the number of available minima down to $7 \pm 2$ as empirically observed. 
This is a very sensitive calculation. If $N$ were a factor of 10 larger, or if $\tau \bar{L}\left(\ll \bar{M} \gg_{p}\right) \sim 0.1$, then $t_{v p}$ is on the order of hours instead of seconds, becoming unrealistic for STM durations. Oppositely, if $t_{v p}$ were much smaller, i.e., less than $\sim 5 \tau$, this would be inconsistent with empirical time scales necessary for formation of any memory trace [24]. In this context, also note that $F^{G}$ scales as $\left(N^{*} N\right)^{1 / 2}$, demanding that both macrocolumnar divergence and minicolumnar convergence of mesocolumnar firings be tested by these calculations.

These results pose serious problems for other models, such as "mean-field" theories or reductionist doctrines. The mean-field approach essentially sets $N=1$ and $N^{*}$ is effectively taken by some investigators to be $\sim 10^{5}$, the size of a macrocolumn, but others even consider it to be as large as $10^{10}$, the total number of neurons in neocortex. The reductionist doctrine claims that only circuitries among a few to several neurons are responsible for neocortical function, and this effectively sets $N \approx N^{*}$, on the order of a few neurons. It is hard to understand how both the capacity and duration of STM can be explained by these other models, even assuming they were or could be derived with realistic synaptic interactions and correct statistical dynamics.

\section{Nearest-neighbor interactions}

It also is of interest to investigate the $\mathrm{NN}$ interactions, e.g., to determine if coefficients of $\left(\nabla M^{G}\right)^{2}$ in $\underline{L}$ lead to excitation or inhibition of similar patterns of firings in nearby mesocolumns.

$\mathrm{NN}$ interactions are repulsive if $\left(\nabla M^{G}\right)^{2}$ contributions are $-\left(\nabla M^{E}\right)^{2}$ or $+\left(\nabla M^{I}\right)^{2}$ in $\underline{L}$. I.e., neighboring mesocolumns tend to fire differently, sharpening the pattern of each mesocolumn. This would be useful for memories contained in a single mesocolumn. If $\left(\nabla M^{G}\right)^{2}$ contributions are attractive, $+\left(\nabla M^{E}\right)^{2}$ or $-\left(\nabla M^{I}\right)^{2}$ in $\underline{L}$, neighboring mesocolumns tend to fire the same. This might facilitate the formation or chunking of larger patterns encompassing several to many mesocolumns.

It should be noted that in mature neocortex, other structures most likely influence NN interactions. Therefore, NN terms as derived here, while possibly being a component of these interactions, may not be the full NN interactions. In any case, note that $\left(\rho \nabla M^{G}\right)^{2}$ and $\tau \dot{M}^{G}$ contributions, a product of their coefficients and gradual spatial-temporal variations of $M^{G}$, are small compared to values of $\bar{L}$ at peaks [7], so that these minima persist in the presence of spatial-temporal fluctuations.

-- Figure 6 --

Figure 6 illustrates contours of the coefficients of $\left(\nabla M^{G}\right)^{2}$ for model $\mathrm{BC}^{\prime}$. The $\left(\nabla M^{E}\right)^{2}$ coefficients are positive and relatively large, $\sim 0.03$, along the main line of the minima in Figure 3(b). The coefficients of $\left(\nabla M^{I}\right)^{2}$ are also positive, and are closer to zero along this line of the minima. The net result is an attractive NN interaction, supporting a scenario of several mesocolumns encoding STM. Note that for other variations of synaptic parameters, this circumstance is not necessarily a typical result. For example, other synaptic parameters yield repulsive NN interactions which may be a contributory mechanism during columnar development [7].

\section{CONCLUSION}

A detailed derivation has been given of spatial-temporal properties of stationary minima of mesocolumnar neocortical firing patterns. The numerical results, i.e., number of clear minima, their likely periods of simultaneous interaction, and nearest-neighbor columnar interactions, all coincide with empirical observations. More detailed calculations will be done: using wider ranges of possible synaptic and columnar parameters, including more laminar circuitry, and testing more global estimates of the time of first passage.

Thus, aspects of conscious experience are derived from neuronal firing patterns, using modern methods of nonlinear nonequilibrium statistical mechanics to develop realistic explicit synaptic interactions. 


\section{ACKNOWLEDGMENTS}

Calculations and plots were facilitated with the PDP-10 MACSYMA Consortium algebraic manipulator at the Massachusetts Institute of Technology, supported in part by USERDA E(11-1)-3070 and NASA NSG 1323. Text preparation was facilitated with the PDP-11/70 CATT UNIX system at the University of California at San Diego. This project has been supported entirely by personal contributions to Physical Studies Institute and to the University of California at San Diego Physical Studies Institute agency account through the Institute for Pure and Applied Physical Sciences.

\section{APPENDIX}

At least to establish notation and key equations, an outline of the derivation of the statistical mechanics of neocortical interactions is given $[6,7]$.

\section{Microscopic neurons}

The microscopic probability $p_{\sigma_{j}}$ for neuron $j$ firing $\left(\sigma_{j}=+1\right.$ if $j$ fires, $\sigma_{j}=-1$ if it does not $)$ is derived from folding a process $\Psi$ for the distribution of $q$ chemical quanta transmitted across a synaptic cleft, with a Gaussian process $\Gamma$ for the distribution of the net effect of postsynaptic interaction as it affects the electrical activity at the axonal trigger zone. Each quanta contains thousands of molecules of neurotransmitter. The probability $p_{\sigma_{j}}$ is essentially the same for $\Psi$ taken to be a Poisson or a Gaussian distribution [6]. For a Poisson-distributed $\Psi$, the mean efficacy is given as

$$
a_{j k}^{*}=\frac{1}{2} A_{j k}^{*}\left(\sigma_{k}+1\right)+B_{j k}^{*},
$$

where $A_{j k}^{*}$ is the activity induced if the presynaptic neuron $k$ fires, and $B_{j k}^{*}$ is a spontaneous background. $A_{j k}^{*}$ and $B_{j k}^{*}$ are on the order of $0.001-0.01$, and for a "typical" neuron there may be as many as $N^{*} \sim 10^{4}-10^{5}$ presynaptic neurons, most emanating locally from within the the range of a macrocolumn of spatial extent $\sim 1 \mathrm{~mm}$. The distribution $\Gamma$ has mean $q v_{j k}$ and variance $\sqrt{q} \phi_{j k}$, where $v_{j k}$ and $\phi_{j k}$ are the net electrical potential and its variance, resp., at the trigger zone; $\left|v_{j k}\right|$ and $\phi_{j k}$ are $\sim 0.1 \mathrm{mV}$, where $v_{j k}$ is positive for excitatory interactions and is negative for inhibitory interactions. Neuron $j$ most likely fires if the threshold potential $V_{j}$ is exceeded within a neuronal relaxation time of $\tau_{n} \sim 5-10 \mathrm{msec} . p_{\sigma_{j}}$ is derived to be

$$
\begin{aligned}
& p_{\sigma_{j}} \approx \frac{\exp \left(-\sigma_{j} F_{j}\right)}{\exp F_{j}+\exp \left(-F_{j}\right)}, \\
& F_{j}=\frac{V_{j}-\sum_{k} a_{j k}^{*} v_{j k}}{\left[\pi \sum_{k^{\prime}} a_{j k^{\prime}}^{*}\left(v_{j k^{\prime}}+\phi_{j k^{\prime}}\right)\right]^{1 / 2}} .
\end{aligned}
$$

\section{Mesoscopic domains}

A mesoscopic probability distribution $P$ is developed for an afferent minicolumn of $N \sim 10^{2}$ neurons, with spatial extent $\rho \sim 10^{2} \mu \mathrm{m}$ and temporal relaxation $\tau \geq \tau_{n}$, having excitatory $(E)$ firing $M^{E}$ and inhibitory $(I)$ firing $M^{I},-N^{G} \leq M^{G} \leq N^{G}$, where $G=E$ or $I . P$ is a response to efferent input within the extent of a macrocolumn of $N^{*}$ neurons. As minicolumns are sensitive to one to several neuronal afferents within $\tau_{n}$, the relaxation time $\tau$ for mesocolumns is of the same order as the relaxation time $\tau_{n}$ for neurons. $E$ and $I$ type neurons have chemically independent synaptic interactions in neocortex, although the firing of a neuron is affected by the contribution from $\bar{G}=E$ and $I$ neurons. A mesocolumn is defined as this afferent minicolumn and efferent macrocolumn scaled down to minicolumnar size, expressing the convergence and divergence of neocortical interactions. The extreme efferent-to-afferent divergence of neocortex, $N^{*}: N$, enables efficient columnar spatial interactions within only 1 to few $\tau_{n}$ periods. Nearest-neighbor (NN) mesocolumnar interactions are defined by overlapping efferent macrocolumnar domains, with centers offset within the extent of a minicolumn. The net effect is 
to average over the $j k$ neurons, yielding $G G^{\prime}$ columnar interactions. To date, no account has been taken of columnar interactions arising from more specific circuitry among the six laminae stratifying all columns. The efferent scaling of $M^{* G}$ macrocolumnar efferent firings to $M^{G}=\left(N / N^{*}\right) M^{* G}$ is done for convenience only, not affecting algebra or numerics, by simultaneously scaling $A_{j k}^{*} \rightarrow\left(N / N^{*}\right) A_{j k}$ and $B_{j k}^{*} \rightarrow\left(N / N^{*}\right) B_{j k}$.

$$
\begin{aligned}
& P=\prod_{G} P^{G}\left[M^{G}(r ; t+\tau) \mid M^{\bar{G}}\left(r^{\prime} ; t\right)\right] \\
& =\sum_{\sigma_{j}} \delta\left(\sum_{j E} \sigma_{j}-M^{E}(r ; t+\tau)\right) \delta\left(\sum_{j I} \sigma_{j}-M^{I}(r ; t+\tau)\right) \prod_{j}^{N} p_{\sigma_{j}} \\
& \approx \prod_{G}\left(2 \pi \tau g^{G G}\right)^{-1 / 2} \exp \left(-N \tau \underline{L}^{G}\right), \\
& \underline{L}^{G}=\left(\dot{M}^{G}-g^{G}\right)^{2} /\left(2 N g^{G G}\right)+M^{G} J_{G} /(2 N \tau)-\underline{V}^{\prime}, \\
& \underline{V}^{\prime G} \rightarrow \sum_{G^{\prime}} \underline{V}_{G^{\prime}}^{\prime G}\left(\rho \nabla M^{G^{\prime}}\right)^{2}, \\
& {\underline{V^{\prime}}}^{G}=-\left(2 N g^{G G}\right)^{-1} g^{G}\left(g^{G}+2 M^{G} / \tau\right) d F^{G}, \\
& g^{G}=-\tau^{-1}\left(M^{G}+N^{G} \tanh F^{G}\right), \\
& g^{G G}=\tau^{-1} N^{G} \operatorname{sech}^{2} F^{G}, \\
& F^{G}=\frac{\left[V^{G}-\sum_{G^{\prime}} a_{G^{\prime}}^{G} v_{G^{\prime}}^{G} N^{G^{\prime}}-\sum_{G^{\prime}} \frac{1}{2} A_{G^{\prime}}^{G} v_{G^{\prime}}^{G} M^{G^{\prime}}\right]}{\left(\pi \sum_{G^{\prime}}\left[\left(v_{G^{\prime}}^{G}\right)^{2}+\left(\phi_{G^{\prime}}^{G}\right)^{2}\right]\left(a_{G^{\prime}}^{G} N^{G^{\prime}}+\frac{1}{2} A_{G^{\prime}}^{G} M^{G^{\prime}}\right)\right)^{1 / 2}}, \\
& a_{G^{\prime}}^{G}=\frac{1}{2} A_{G^{\prime}}^{G}+B_{G^{\prime}}^{G},
\end{aligned}
$$

where $J_{G}$ are constraints on $M^{G}$ from long-ranged fibers.

The NN differential interactions are further specified as

$$
\begin{aligned}
d F^{G}= & -\tanh F^{G}\left(f_{n}^{*} d F_{1}^{G}-2 f_{n}^{* 2} d F_{2}^{G} \tanh F^{G}\right), \\
d F_{1}^{G}= & \rho N^{G} \sum_{G^{\prime} z} F_{, G^{\prime}}^{G} M_{: z}^{G^{\prime}} \hat{\varepsilon}_{z} \\
& +\frac{1}{2} \rho^{2} N^{G} \sum_{G^{\prime} G^{\prime \prime} z z^{\prime}} F_{, G^{\prime} G^{\prime \prime}}^{G} M_{: z}^{G^{\prime}} M_{: z^{\prime}}^{G^{\prime \prime}} \hat{\varepsilon}_{z} \hat{\varepsilon}_{z^{\prime}} \\
& +\frac{1}{2} \rho^{2} N^{G} \sum_{G^{\prime} z z^{\prime}} F_{, G^{\prime}}^{G} M_{: z z^{\prime}}^{G^{\prime}} \hat{\varepsilon}_{z} \hat{\varepsilon}_{z^{\prime}}, \\
d F_{2}^{G}= & \rho^{2} N^{G} \sum_{G^{\prime} G^{\prime \prime} z z^{\prime}} F_{, G^{\prime}}^{G} F_{, G^{\prime \prime}}^{G} M_{: z}^{G^{\prime}} M_{: z^{\prime}}^{G^{\prime \prime}} \hat{\varepsilon}_{z} \hat{\varepsilon}_{z^{\prime}},
\end{aligned}
$$




$$
\begin{aligned}
& {[\cdots]_{: z} \equiv \frac{\partial[\cdots]}{\partial z}=\hat{\varepsilon}_{\hat{z}} \cdot \nabla_{r}[\cdots],} \\
& {[\cdots]_{, G} \equiv \frac{\partial[\cdots]}{\partial M^{G}},} \\
& \hat{\varepsilon}=\frac{\varepsilon}{|\varepsilon|}=\frac{\left(r-r^{\prime}\right)}{\left|r-r^{\prime}\right|}, \\
& r=(x, y), \quad z=\{x, y\}, \\
& \dot{M}^{G}(t)=\tau^{-1}\left[M^{G}(t+\tau)-M^{G}(t)\right], \\
& \nabla M^{G}(x, y)=\rho^{-1}\left[M^{G}(x+\rho, y)-M^{G}(x, y)\right] \hat{x} \\
& \quad+\rho^{-1}\left[M^{G}(x, y+\rho)-M^{G}(x, y)\right] \hat{y} .
\end{aligned}
$$

An integration by parts is performed in the $M_{: z z^{\prime}}^{G^{\prime}} \mathrm{NN}$ terms to give a form $\left(\nabla M^{G}\right)\left(\nabla M^{G^{\prime}}\right)$. The numerical factor $f_{n}^{*}$ depends on the spatial dimension $n$, and arises from considering $\sum_{j} \sum_{k \neq j}$ in $F_{j}$ to establish how much overlap all NN domains at $r^{\prime} \leq r+\rho$ make with domain $r$ : If $N^{*}$ were to equal $N$, e.g., as in the mathematically similar frustrated magnetic system [18], $f_{1}=1 / 4=0.25$, $f_{2}=(3 / 4)^{3 / 2} \pi^{-1}=0.207, f_{3}=17 / 64=0.266$. (Of course, the amount of overlap per nearest-neighbor $f_{n} / n_{\mathrm{NN}}$ decreases as $n$ increases from 1 to 2 to 3 , where $n_{\mathrm{NN}}$ is the number of NN.)

For neocortex, $f_{2}^{*} \approx(2 / 3 \pi)\left(N / N^{*}\right)^{1 / 2}=0.212\left(N / N^{*}\right)^{1 / 2} \sim 0.00671$ should appear in $d F^{G} . f_{2}^{*}$ appears instead of $f_{2}$ because in this problem the double sum to be considered is $\sum_{j=1}^{N} \sum_{k=1}^{N^{*}}$, where $\sum_{j}^{N}=\frac{1}{2} \pi \rho^{2}$ and $N^{*} \approx 10^{3} N$. For isotropically interacting domains with spatially uncorrelated species $E$ and $I$, contributions to $\tilde{L}$ from the linear term in $\hat{\varepsilon}_{z}$ average to zero, and

$$
\sum_{z z^{\prime}} \int d^{n} r M_{: z}^{G^{\prime}} M_{: z^{\prime}}^{G^{\prime \prime}} \hat{\varepsilon}_{z} \hat{\varepsilon}_{z^{\prime}}=\frac{1}{n} \sum_{z} \int d^{n} r \delta_{G^{\prime \prime}}^{G^{\prime}} M_{: z}^{G^{\prime}} M_{: z}^{G^{\prime \prime}}
$$

The number of neuronal parameters may be significantly reduced, without drastically altering the conclusions of these studies, by further averaging of $G G^{\prime}$ and setting $A_{G^{\prime}}^{G}, B_{G^{\prime}}^{G}, v_{G^{\prime}}^{G}=v^{G}, \phi_{G^{\prime}}^{G}=\phi^{G}$, all to be considered as static with respect to time scales used for STM calculations. As discussed previously [7], these parameters do change when LTM is considered. Then, $F^{G}$ is reduced to

$$
\begin{aligned}
& F^{G}=\beta^{G}\left(\gamma^{G}-\alpha^{G} M^{-}\right) /\left(1+\alpha^{G} M^{+}\right)^{1 / 2}, \\
& \alpha^{G}=N^{* G} A^{* G} /\left(2 N^{*} N^{G} a^{* G}\right) \ll 1, \\
& a^{* G}=\frac{1}{2} A^{* G}+B^{* G}, \\
& \beta^{G}=\left[N^{*} a^{* G}\left[1+\left(\phi^{G} / v^{G}\right)^{2} \pi\right]^{-1}\right]^{1 / 2}<N^{* 1 / 2}, \\
& \gamma^{G}=V^{G} /\left(a^{*} v^{G} N^{*}\right)-N^{*-} / N^{*}, \\
& M^{-}=M^{E}-M^{I}, M^{+}=M^{E}+M^{I}, \\
& N^{*-}=N^{* E}-N^{* I}, N^{*} \equiv N^{*+}=N^{*} E+N^{* I} .
\end{aligned}
$$

For neocortex, it is found that $\left|\gamma^{G}\right| \sim 0.1, \alpha^{G} \sim 0.005$ and $\beta^{G} \sim 5$. 
[Note that this calculation corrects previous papers [6,7], which made an estimate of NN in two dimensions, but which neglected the $\left(N / N^{*}\right)^{1 / 2}$ factor in $f_{2}^{*}$. Apart from errors in writing $\frac{1}{2} \beta^{G}$ instead of $\beta^{G}$ in $d F_{1}^{G}$, and in an extra factor of $\frac{1}{2}$ in $d F_{2}^{G}$, these changes are not large enough to invalidate any conclusions of that study. The net effect is to correct the most important term in Eq. (2.2) of Ref7. with $d F_{1}^{G}$ proportional to $\beta^{G}\left(\nabla^{2} M^{-}\right)$by a factor of $12 f_{2}^{*} \approx 0.0805$. A salutary effect is to lower the propagation velocity of the dispersion relations derived from the Euler-Lagrange equation to $\sim 1 \mathrm{~cm} / \mathrm{sec}$, taking typical wave numbers to correspond to macrocolumnar distances of $\sim 30 \rho$. Calculated frequencies are on the order of EEG frequencies $\sim 10^{2} \mathrm{sec}^{-1}$. These mesoscopic propagation velocities permit processing of mesoscopic interactions of several minicolumns within $\sim 10^{-1} \mathrm{~cm}$, simultaneous with processing of macroscopic interactions over tens of centimeters via association fibers with macroscopic propagation velocities $\sim 600-900 \mathrm{~cm} / \mathrm{sec}$. I.e., both can occur within $\sim 10^{-1} \mathrm{sec}$.]

\section{Macroscopic regions}

As calculated above to resolution $\tau$, firings of $M^{G}(t+\theta)$ for $0 \leq \theta \leq \tau$ arise due to interactions within memory $\tau$ as far back as $M^{G}(t+\theta-\tau)$. I.e., firings of afferents $M^{G}(t+\tau)$ at time $t+\tau$ have been calculated from interactions $M^{G}(t)$ at the $\tau$-averaged efferent firing time $t$. With equal likelihood throughout time $\tau$, any of the $N^{*}$ uncorrelated efferent neurons can contribute to change the mesocolumnar mean firings and fluctuations of their $N$ uncorrelated afferents. Therefore, for $\theta \leq \tau$, at least to resolution $\theta \geq \tau / N$ and to order $\theta / \tau$, it is reasonable to assume that efferents effect a change in afferent mean firings of $\theta \dot{M}^{G}=M^{G}(t+\theta)-M^{G}(t) \approx \theta g^{G}$ with variance $\theta g^{G G}$. Indeed, columnar firings (e.g., as measured by averaged evoked potentials) are observed to be faithful continuous probabilistic measures of individual neuronal firings (e.g., as measured by poststimulus histograms) [25]. Defining $P^{\theta}=P\left[M^{G}(r ; t+\theta) \mid M^{G}\left(r^{\prime} ; t\right)\right]$ as a Gaussian distribution similar to $P^{\tau}=P\left[M^{G}(r ; t+\tau) \mid M^{G}\left(r^{\prime} ; t\right)\right]$ in Eq. (A3a), $P^{\theta}$ satisfies the Markovian Chapman-Kolmogorov equation $P^{\theta+\theta^{\prime}}=\int P^{\theta} P^{\theta^{\prime}}$, consistent with considering $P^{\tau}$ to be Markovian and as evolving from $P^{\theta}$. It is thereby conjectured that requiring $M^{G}$ to be continuous at this resolution, albeit not necessarily differentiable, and prepoint discretized $P^{\tau}$ to be Markovian, suffice to reasonably define $P^{\theta}$ at the mesoscopic scale for $\theta \leq \tau$. E.g., the same result should be approximately obtained if mesoscopic distributions $P^{\theta}$ of variables $M^{G}$ were extracted after considering microscopic $\sigma_{j}$ contributions to $p_{\sigma_{j}}$ to have a temporal distribution within $\tau$, e.g., Poisson, still respecting postsynaptic delays $\sim \tau_{n} \leq \tau$. This argument permits construction of a path integral at a finer resolution $\theta \ll \tau$, although a resolution of $\theta \approx \tau$ would suffice for this study.

The main problem addressed by this theory is to reasonably extract the mesoscopic variables from the microscopic ones, in a form sufficiently tractable for further macroscopic development. The macroscopic probability $\tilde{P}\left[\tilde{M}(t) \mid \tilde{M}\left(t_{0}\right)\right]$ is developed by folding $\Lambda \sim 5 \times 10^{5}$ mesocolumns of spatial extent $\Omega \approx 5 \times 10^{9} \mu \mathrm{m}^{2}$, labeled by $v(v=\Lambda+1 \equiv 1)$, and folding the differential propagator $P^{\theta}$ for $u+1$ time periods, spanning time $t-t_{0}=s \theta$, each period of duration $\theta \leq \tau$ labeled by $s$. With boundary conditions $\tilde{M}_{u+1}=\tilde{M}(t)$ and $\tilde{M}_{0}=\tilde{M}\left(t_{0}\right)$, and defining $\tilde{M}=\left\{M^{G v}\right\}$,

$$
\begin{aligned}
& \tilde{P}\left[\tilde{M} \mid \tilde{M}_{0}\right]=\int \cdots \int \underline{D} \tilde{M} \exp (-N \tilde{S}) \delta(\tilde{M}=\tilde{M}(t)) \delta\left(\tilde{M}_{0}=\tilde{M}\left(t_{0}\right)\right) \\
& \tilde{S}=\int_{t_{0}}^{t} d t^{\prime} \tilde{L} \equiv \theta \sum_{s=1}^{u+1} \tilde{L} \\
& \tilde{L}=\Lambda \Omega^{-1} \int d^{2} r \underline{L} \equiv 1 \sum_{v=1}^{\Lambda} \underline{L} \\
& \underline{L}=\underline{L}^{E}+\underline{L}^{I}=\frac{1}{2 N}\left(\frac{1}{\theta}\left(M_{s+1}^{G v}-M_{s}^{G v}\right)-g_{s}^{G v}\right)
\end{aligned}
$$




$$
\begin{array}{r}
\times g_{G G^{\prime} s}^{v}\left(\frac{1}{\theta}\left(M_{s+1}^{G^{\prime} v}-M_{s}^{G^{\prime} v}\right)-g_{s}^{G^{\prime} v}\right) \\
+\frac{1}{2 N \tau} M_{s}^{G v} J_{G s}^{v} \\
-\rho^{2} \sum_{G}{\underline{V^{\prime \prime}}}_{G^{\prime}}^{G}\left(M_{s}^{G^{\prime} v+1}-M_{s}^{G^{\prime} v}\right)^{2}, \\
\underline{D} \tilde{M}=\prod_{v=1}^{\Lambda} \prod_{G}^{E, I} \prod_{s=1}^{u+1}\left[(2 \pi \theta)^{-1 / 2}\left(g_{s}^{v}\right)^{1 / 4}\right] \prod_{s^{\prime}=1}^{u} d M_{s^{\prime}}^{G v}, \\
g_{s}^{v}=\left\|g_{G G^{\prime} s}^{v}\right\|=\operatorname{det}\left(g_{G G^{\prime} s}^{v}\right)=g_{E E s}^{v} g_{I I s}^{v}, \\
g_{G G^{\prime} s}^{v}=\left(g_{s}^{G G^{\prime} v}\right)^{-1},
\end{array}
$$

where the Einstein convention of summing over factors with repeated $G$ indices is henceforth assumed, except when vertical bars appear on an index, e.g., $|G|$.

The prepoint discretization of $\underline{L}(M), \theta \dot{M}^{G}\left(t^{\prime}\right) \rightarrow M_{s+1}^{G}-M_{s}^{G}$ and $M^{G}\left(t^{\prime}\right) \rightarrow M_{s}^{G}$, is derived from the biophysics of neocortex. This is not equivalent to the Stratonovich midpoint discretization of a proper Feynman Lagrangian $\underline{L}_{F}, \theta \dot{M}^{G}\left(t^{\prime}\right) \rightarrow M_{s+1}^{G}-M_{s}^{G}$ and $M^{G}\left(t^{\prime}\right) \rightarrow \frac{1}{2}\left(M_{s+1}^{G}+M_{s}^{G}\right)$ [9]. The discretization and the Lagrangian (and $g$ ) must be defined consistently to give an invariant scalar $g^{1 / 2} P[M]$. The covariant Feynman Lagrangian is defined in terms of a stationary principle, and the transformation to the Stratonovich discretization permits the use of the standard calculus.

$$
\begin{aligned}
& \tilde{S}_{F}=\min \Lambda \Omega^{-1} \int d t^{\prime} \int d^{2} r \underline{L}_{F}, \\
& \underline{L}_{F}=\frac{1}{2} N^{-1}\left(\dot{M}^{G}-h^{G}\right) g_{G G^{\prime}}\left(\dot{M}^{G^{\prime}}-h^{G^{\prime}}\right)-\underline{V}, \\
& h^{G}=g^{G}-\frac{1}{2} g^{-1 / 2}\left(g^{1 / 2} g^{G G^{\prime}}\right)_{G^{\prime}}, \\
& \underline{V}=\underline{V}^{\prime}-\left(\frac{1}{2} h_{; G}^{G}+R / 6\right) / N, \\
& \underline{V}^{\prime}=\underline{V}^{\prime E}+\underline{V}^{\prime}-M^{G} J_{G} /(2 N \tau), \\
& h_{; G}^{G}=g^{-1 / 2}\left(g^{1 / 2} h^{G}\right)_{, G},
\end{aligned}
$$

where "min" specifies that the short-time propagator is evaluated by expanding about that path which makes the action $\tilde{S}_{F}$ stationary. The Riemannian curvature $R$ arises from the nonlinear inverse variance $g_{G G^{\prime}}$, which is a bona fide metric of this parameter space,

$$
\begin{aligned}
R= & g^{-1}\left(g_{E E, I I}+g_{I I, E E}\right) \\
& -\frac{1}{2} g^{-2}\left[g_{I I}\left(g_{E E, E} g_{I I, E}+g_{E E, I}^{2}\right)\right. \\
& \left.+g_{E E}\left(g_{I I, I} g_{E E, I}+g_{I I, E}{ }^{2}\right)\right] .
\end{aligned}
$$

It also should be noted that the Feynman Lagrangian does not define the most-probable short-time propagator, in the sense of a WKB expansion, when $R \neq 0$ [9]. (When comparing the short-time propagators $P_{F}$ and $P_{\mathrm{WKB}}$, an $R / 12$ first-order WKB correction appears in $\underline{L}_{\mathrm{WKB}}$ instead of $R / 6$ in $L_{F}$, and the prefactor of the exponential in $P_{\mathrm{WKB}}$ includes the van Vleck determinant, $\propto g^{1 / 2}$, multiplied by 
$g^{1 / 4} \propto 1+R / 12$, which essentially accounts for the difference between $L_{F}$ and $L_{\mathrm{WKB}}$.) However, for the system of interest here, the scalar Feynman Lagrangian is more useful because of its variational principle.

\section{Other representations}

To first order in $\left(\nabla M^{G}\right)^{2}$, the differential evolution of $\tilde{P}$ corresponding to Eq. (A6) or Eq. (A7) is given by the Schrodinger-type equation

$$
\begin{gathered}
\frac{\partial \tilde{P}}{\partial t}=\Omega^{-1} \int d^{2} r\left[\frac{1}{2} \hat{\delta}_{G} \hat{\delta}_{G^{\prime}}\left(g^{\prime G G^{\prime}} \tilde{P}\right)-\hat{\delta}_{G}\left(g^{\prime G} \tilde{P}\right)\right] \\
\approx \Omega^{-1} \int d^{2} r\left[\frac{1}{2}\left(g^{G G^{\prime}} \tilde{P}\right)_{, G G^{\prime}}-\left(g^{G} \tilde{P}\right)_{, G}+N \underline{V}^{\prime} \tilde{P}\right], \\
\tau g^{\prime G}=\tau g^{G}-d F^{|G|} N^{|G|} \tanh F^{|G|}, \\
\tau g^{\prime G G^{\prime}}=\tau g^{G G^{\prime}}+\delta_{G}^{G^{\prime}} d F^{G} N^{G} \operatorname{sech}^{2} F^{G}, \\
\hat{\delta}_{G}[\cdots]=\frac{\delta\left(\int d^{2} r^{\prime}[\cdots]\left(M^{G^{\prime}}, \nabla^{\prime} M^{G^{\prime}}, \nabla^{\prime 2} M^{G^{\prime}}\right)\right)}{\delta M^{G}(r)} \\
=[\cdots]_{, G}-\nabla_{z}[\cdots]_{, \nabla_{z} G}+\nabla_{z}^{2}[\cdots]_{, \nabla_{z}{ }^{2} G} \\
\equiv[\cdots]_{, G}-[\cdots]_{, G_{: z}: z}+[\cdots]_{G_{: z z}: z z}, \\
{[\cdots]_{, G_{: z}: z}=[\cdots]_{, G_{: z} G^{\prime}} M^{G^{\prime}}: z} \\
+[\cdots]_{G_{: z} G_{: z}^{\prime}} M^{G^{\prime}}: z z
\end{gathered}
$$

where the Einstein convention is extended to $z$ indices. The Fokker-Planck functional differential equation, i.e., with respect to $\hat{\delta}_{G}$ and possessing no potential term, corresponds to the derivation of NN interactions in $\underline{L}$ [6]. However, the simpler partial differential equation arises from expansion of the $\nabla M^{G}$ perturbations, yielding a Schrodinger-type equation with a $V^{\prime}$ potential of $\mathrm{NN}$ interactions.

A Hamiltonian operator $\hat{\underline{H}}$ and commutation relations are derived by defining an evolution operator $U\left(t, t_{0}\right)[9]$. In a firing state basis $\mid M^{G}>$,

$$
\begin{aligned}
& \tilde{P}\left[\tilde{M} \mid \tilde{M}_{0}\right]=<\tilde{M}\left|\tilde{U}\left(t, t_{0}\right)\right| \tilde{M}_{0}> \\
& i \frac{\partial \tilde{U}\left(t, t_{0}\right)}{\partial t}= \tilde{\tilde{H}} \tilde{U}\left(t, t_{0}\right) \equiv \Omega^{-1} \int d^{2} r \hat{H} \prod_{V} U\left(t, t_{0}\right), \\
& \hat{H}(\hat{p}, \hat{M})=-\frac{i}{2} \hat{p}_{G} \hat{p}_{G^{\prime}} g^{G G^{\prime}}(\hat{M})+\hat{p}_{G} g^{G}(\hat{M}) \\
&+i N \underline{V}^{\prime}(\hat{M}), \\
& \hat{M}^{G} \mid M^{G}>= M^{G} \mid M^{G}>, \\
& \hat{p}_{G} \mid M^{G}>=-i \partial / \partial M^{G} \mid M^{G}>, \\
& {\left[\hat{M}^{G}, \hat{p}_{G^{\prime}}\right]=} i \delta_{G^{\prime}}^{G}, \\
& {\left[\hat{M}^{G}, \hat{M}^{G^{\prime}}\right]=\left[\hat{p}_{G}, \hat{p}_{G^{\prime}}\right]=0, }
\end{aligned}
$$




$$
\begin{aligned}
& \underline{\hat{p}}_{G}(t)=U^{-1}(t) \hat{p}_{G} U(t), \\
& \hat{\underline{M}}^{G}(t)=U^{-1}(t) \hat{M}^{G} U(t), \\
& U(t) \equiv U(t, 0)=\left[U^{\backslash(d g}(t)\right]^{-1}, \\
& \underline{\hat{\hat{p}}}_{G}=i\left[\hat{\hat{H}}(\underline{\hat{p}}, \hat{\hat{M}}), \underline{\hat{p}}_{G}\right], \\
& \dot{\hat{M}}^{G}=i\left[\hat{\hat{H}}(\underline{\hat{p}}, \hat{\underline{M}}), \hat{\hat{M}}^{G}\right] .
\end{aligned}
$$

From this representation, with the use of $U(t+\theta, t) \approx 1-i \theta \hat{H}+O\left(\theta^{2}\right),\left\langle M^{\prime} \mid M\right\rangle=\delta\left(M^{\prime}-M\right)$, and $<M|p\rangle=(2 \pi)^{-1} \exp \left(i p_{G} M^{G}\right),(M, p)$-phase-space path-integral representations can be derived in terms of Hamiltonian functions $\underline{H}(p, M)$ corresponding to the previous Lagrangian functions $\underline{L}(\dot{M}, M)$ in the $M$-coordinate basis [9].

The Langevin rate equations, with $M^{G} J_{G}$ simulated by boundary conditions, in the Stratonovich representation, are

$$
\begin{aligned}
& \dot{M}^{G}=g^{\prime G}-\frac{1}{2} \delta^{j k} \hat{g}_{j}^{G^{\prime}} \hat{g}_{k, G^{\prime}}^{G}+\hat{g}_{j}^{G} \eta_{j}, \\
& \hat{g}_{j}^{G} \hat{g}_{k}^{G^{\prime}} \delta^{j k}=g^{\prime G G^{\prime}} / \tau, \\
& <\eta_{i}(t)>=0, \\
& <\eta_{i}(t) \eta_{j}\left(t^{\prime}\right)>=\delta_{i j} \delta\left(t-t^{\prime}\right),
\end{aligned}
$$

where $\eta_{j}$ represents Gaussian white noise arising from the microscopic neuronal system labeled by $j$, and the Einstein convention is extended to $j k$ indices. Because of these derivations, it is reasonable to take $\hat{g}_{j}^{G} \approx \delta_{G^{\prime}}^{G}\left[g^{\prime G G^{\prime}} /\left(N^{G} \tau\right)\right]^{1 / 2}, j=1, \cdots, N$, and $j \in G ; \hat{g}_{j}^{G}=0, j \in G^{\prime} \neq G$.

The "information" contained in this description is well defined as

$$
\hat{\Upsilon}[\tilde{P}]=\int \cdots \int \underline{D} \tilde{M} \tilde{P} \ln (\tilde{P} / \bar{P}),
$$

where $\bar{P}$ is a reference stationary state. Although many microscopic synaptic degrees of freedom have been averaged over, many degrees of freedom are still present, as measured by $d M_{s}^{G V}$ [7].

Another common set of equations derived from the other above differential equations consists of the Euler-Lagrange variational equations associated with $\underline{L}_{F}$. The minimum (extrema) condition leads to a set of 12 coupled first-order differential equations, with coefficients nonlinear in $M^{G}$, in the 12 variables $\left\{M^{G}, \dot{M}^{G}, \ddot{M}^{G}, \nabla M^{G}, \nabla^{2} M^{G}\right\}$ in $(r ; t)$ space. In the neighborhood of extrema $\ll \bar{M}^{G} \gg, \underline{L}_{F}$ can be expanded as a Ginzburg-Landau polynomial. To investigate first-order linear oscillatory states, only powers up to 2 in each variable are kept, and from this the variational principle leads to a relatively simple set of coupled linear differential equations with constant coefficients:

$$
\begin{aligned}
& 0=\hat{\delta} \underline{L}_{F}=\underline{L}_{F, \dot{G}: t}-\hat{\delta}_{G} \underline{L}_{F} \\
& \approx-\underline{f}_{|G|} \ddot{M}^{|G|}+\underline{f}_{-G}^{1} \underline{\dot{M}}^{G\urcorner}-\underline{g}_{|G|} \nabla^{2} \underline{M}^{|G|}+\underline{b}_{|G|} \mid \underline{M}^{|G|}+\underline{b} \underline{M}^{G^{\urcorner}}, \\
& G\urcorner \neq G, \\
& {[\cdots]_{, \dot{G}: t}=[\cdots]_{, \dot{G} G^{\prime}} \dot{M}^{G^{\prime}}+[\cdots]_{, \dot{G} \dot{G}^{\prime}} \ddot{M}^{G^{\prime}},} \\
& \underline{M}^{G}=M^{G}-\ll \bar{M}^{G} \gg, \\
& \underline{f}_{E}^{1}=-\underline{f}_{I}^{1} \equiv \underline{f} .
\end{aligned}
$$


These equations are then Fourier transformed and the resulting dispersion relation is examined to determine for which values of the synaptic parameters $Z$ and of $\underline{\xi}$, the conjugate variable to $r$, can oscillatory states, $\omega(\underline{\xi})$, persist $[6,7]$.

\section{Chaotic behavior and spin-glass analogy}

Several investigators have drawn analogies between neocortical interactions and spin-glasses [26,27]. However, these comparisons have been made considering only simple functional forms for "average" neurons, neglecting the proper development mentioned in the Introduction, that requires consistent realistic treatment of (a) synaptic interactions, (b) columnar statistics, and (c) dynamics of their evolution. Essentially, quadratic Hamiltonians have been assumed without theoretical justification or empirical specificity. A quadratic form would require (unspecified) additional assumptions beyond the centering mechanism in the text and linearization about $M^{G}=0$.

Since it is highly speculative that spin-glass transitions could occur from short-ranged interactions [28], such analogies for neocortex, if they exist, should be confined to the long-ranged interactions, or to possible long-ranged dipole-dipole interactions derived from short-ranged interactions. Columnar interactions often may be considered as dipole-dipole interactions arising from the highly populous vertically oriented pyramidal neurons. E.g., EEG and MEG are often modeled as arising from a dipole layer of cortical tissue [10], the top laminae considered to be prominent sites of excitatory pyramidal efferents interacting with afferent dendrites, and the lower laminae considered to be prominent sites of axonal discharge of pyramidal afferents. However, it is not very plausible that columnar interactions should be considered as having random interactions at mesoscopic resolutions, a condition assumed for spin-glass modeling, even granted that columnar distribution is somewhat askew. In neocortex the long-ranged neuronal interactions appear independent of and highly organized relative to the short-ranged neuronal interactions, so it is unlikely that they could be a source of random interactions to these firing dipoles. Furthermore, for neocortex, it is not necessary to assume a random connectivity of $A_{j k}^{*}$ in Eq. (A1) for the short-ranged synaptic interactions. Although it might be possible to learn about neocortex from spin-glass Hamiltonians, oppositely, reasonable (currently accepted) Hamiltonians or Lagrangians for frustrated ferromagnetic-antiferromagnetic interactions might be developed into nonlinear nonequilibrium statistical magnetic domain-interactions, using the mathematical formalism being applied to neocortex [18].

Other investigators have also made analogies between spin-glass and neocortical systems [27], drawing conclusions regarding chaotic behavior from the simple functional mean-field form

$$
m^{G}=\tanh \left[\bar{a}+\bar{b}\left(m^{E}-m^{I}\right)\right],
$$

where $m^{G}=M^{G} / N$ here. In addition to the difficulties mentioned in making these analogies, Eq. (A12) corresponds to considering all members of a simple Ising-type system approximating neurons or spins interacting with each other, a mean-field limit. Note that for neocortical domains, $\bar{a}$ and $\bar{b}$ scale as $N^{1 / 2}$, and since the mean-field limit requires $10^{5}-10^{10}$ neurons or even more spins for magnetic domains, the tanh function essentially would be a sharp step function in $\left(m^{E}-m^{I}\right)$.

Although there is some evidence for chaotic behavior in neocortex [7], these other studies do not reinforce this possibility regarding Eq. (A12) because this assumed functional form is not derived from any underlying theory, and $\bar{a}$ and $\bar{b}$ are not further specified. Furthermore, this functional form does not appear to be the appropriate limit of this theory given here. However, it can be shown that Eq. (A12) could describe chaotic behavior under some specific assumptions. This demonstration is useful as it gives more insight into possible mechanisms that may occur in neocortex or spin-glass. It first would have to be proven that to study chaotic behavior, measurements most typically should be made at discrete times on the order of $\theta \leq \tau$, and that for some additional reason the system would be required to follow the path of most probable transition states, for a series of transitions, from a fixed prepoint to the most probable postpoint of each transition.

The transition path is developed from the short-time path-integral as [29]

$$
\dot{M}^{G}=g^{G}-\frac{1}{2} g^{1 / 2}\left(g^{-1 / 2} g^{G G^{\prime}}\right)_{G^{\prime}} .
$$


Clearly, this equation is not another representation of deterministic Langevin rate equations, e.g., as derived from Eq. (A9). (Note the order of exponents of the factors of g.) Equation (A13) holds more generally than for just the midpoint discretization if it is rewritten in terms of [29]

$$
\begin{aligned}
& \hat{M}^{G}=M_{i}^{G}+r \Delta^{G}, 0 \leq r \leq 1, \\
& \Delta^{G}=M_{f}^{G}-M_{i}^{G}, \\
& M_{i}^{G}=M^{G}(t), \\
& M_{f}^{G}=M^{G}(t+\theta), \\
& {[\cdots]_{, G}=\partial[\cdots] / \partial M_{f}^{G}=r \partial[\cdots] / \partial \hat{M}^{G} .}
\end{aligned}
$$

Since it is usually desirable for computations to have maps that are easily iterated to study chaotic behavior [30,31], it is most convenient to use the prepoint discretization, $r=0$, whereby the right-hand side of Eq. (A14) is evaluated at $M^{G}(t)$, and the left-hand side is discretized as $\dot{M}^{G}=\theta^{-1}\left[M^{G}(t+\theta)-M^{G}(t)\right]$. I.e., $\dot{M}^{G} \rightarrow g^{G}$, or

$$
M^{G}(t+\theta)=-N^{|G|} \tanh F^{|G|}(t) .
$$

Thus, using Eq. (A14), then, but only then, Eq. (A13) reduces to Eq. (A15). Then the results previously obtained for chaotic behavior for the ansatz of Eq. (A12) are seen to hold quite generally, but for domains of mesocolumnar extent. This is an important clarification, since domains are larger systems than individual spins or neurons, and therefore are more amenable to experimental study. 


\section{REFERENCES}

[1] G.A. Miller, "The magical number seven, plus or minus two," Psychol. Rev. 63, 81-97 (1956).

[2] K.A. Ericsson and W.G. Chase, "Exceptional memory," Am. Sci. 70, 607-615 (1982).

[3] L. Ingber, “Attention, physics and teaching," J. Social Biol. Struct. 4, 225-235 (1981).

[4] L. Ingber, Karate: Kinematics and Dynamics (Unique, Hollywood, CA, 1981).

[5] L. Ingber, “Towards a unified brain theory," J. Social Biol. Struct. 4, 211-224 (1981).

[6] L. Ingber, "Statistical mechanics of neocortical interactions. I. Basic formulation," Physica D 5, 83-107 (1982).

[7] L. Ingber, "Statistical mechanics of neocortical interactions. Dynamics of synaptic modification," Phys. Rev. A 28, 395-416 (1983).

[8] H. Haken, Synergetics, 3rd ed. (Springer, New York, 1983).

[9] F. Langouche, D. Roekaerts, and E. Tirapegui, Functional Integration and Semiclassical Expansions (Reidel, Dordrecht, The Netherlands, 1982).

[10] P.L. Nunez, Electric Fields of the Brain: The Neurophysics of EEG (Oxford University Press, London, 1981).

[11] S. Amari, "Competitive and cooperative aspects in dynamics of neural excitation and selforganization," in Competition and Cooperation in Neural Nets, ed. by S.-I. Amari and M.A. Arbib (Springer, Berlin, 1982), p. 1-28.

[12] J.R. Bergen and B. Julesz, "Parallel versus serial processing in rapid pattern discrimination," Nature 303, 696-698 (1983).

[13] L. Ingber, "Editorial: Learning to learn," Explore 7, 5-8 (1972).

[14] R.M. Shiffrin and W. Schneider, "Controlled and automatic human information processing. II. Perceptual learning, automatic attending, and a general theory.," Psych. Rev. 84, 127-190 (1977).

[15] L. Ingber, "Nuclear forces," Phys. Rev. 174, 1250-1263 (1968).

[16] L. Ingber, "Riemannian corrections to velocity-dependent nuclear forces," Phys. Rev. C 28, 2536-2539 (1983).

[17] L. Ingber, "Path-integral Riemannian contributions to nuclear Schrödinger equation," Phys. Rev. D 29, 1171-1174 (1984).

[18] L. Ingber, "Nearest-neighbor frustrated magnetic domains," PSI Report No. NNFMD-83, Physical Studies Institute, Solana Beach, CA, 1983.

[19] L. Ingber, "Statistical mechanics of nonlinear nonequilibrium financial markets," Math. Modelling 5, 343-361 (1984).

[20] V.B. Mountcastle, "An organizing principle for cerebral function: The unit module and the distributed system," in The Mindful Brain, ed. by G.M. Edelman and V.B. Mountcastle (Massachusetts Institute of Technology, Cambridge, 1978), p. 7-50.

[21] V.B. Mountcastle, R.A. Andersen, and B.C. Motter, "The influence of attentive fixation upon the excitability of the light-sensitive neurons of the posterior parietal cortex," J. Neurosci. 1, 1218-1235 (1981).

[22] G.S. Agarwal and S.R. Shenoy, "Observability of hysteresis in first-order equilibrium and nonequilibrium phase transitions," Phys. Rev. A 23, 2719-2723 (1981).

[23] S. Grossberg, "Associative and competitive principles of learning and development," in Competition and Cooperation in Neural Nets, ed. by S. Amari and M.A. Arbib (Springer, New York, 1982), p. 295-341.

[24] B. Libet, "Brain stimulation in the study of neuronal functions for conscious sensory experience," Human Neurobiol. 1, 235-242 (1982).

[25] E.R. John, "Switchboard versus statistical theories of learning and memory," Science 177, 850-864 (1972). 
[26] J.J. Hopfield, "Neurons with graded responses have collective computational properties like those of two-state neurons," Proc. Natl. Acad. Sci. USA 81, 3088-3092 (1984).

[27] M.Y. Choi and B.A. Huberman, "Dynamic behavior of non-linear networks," Phys. Rev. A 28, 1204-1206 (1983).

[28] D. Bowman and J.W. Halley, "Spin-glass model with short-range and long-range interactions," Phys. Rev. B 25, 1892-1920 (1982).

[29] H. Dekker, "On the most probable transition path of a general diffusion process," Phys. Lett. A 80, 99-101 (1980).

[30] E. Ott, "Strange attractors and chaotic motions of dynamical systems," Rev. Mod. Phys. 53, 655-671 (1981).

[31] J.D. Farmer, "Chaotic attractors of an infinite-dimensional dynamic system," Physica D 4, 366-393 (1982). 


\section{TABLE CAPTIONS}

TABLE I. Tabulated, for values of $\bar{M}^{G}$ spanning the diagonal from $\bar{M}^{G}=-N^{G}$ to $\bar{M}^{G}=N^{G}$, are the corresponding values of the Lagrangian $\tau \overline{\underline{L}}_{\mathrm{BC}}$ and the determinant of its Hessian $\left\|\tau \overline{\underline{L}}_{\mathrm{BC}^{\prime}, G G^{\prime}}\right\|$.

\section{FIGURE CAPTIONS}

FIG. 1. (a) Contours for values less than 1 are drawn for $\tau \bar{L}_{\text {IC }}$. The $\bar{M}^{E}$ axis increases to the right, from $-N^{E}=-80$ to $N^{E}=80$. The $\bar{M}^{I}$ axis increases upwards, from $-N^{I}=-30$ to $N^{I}=30$. The resolution is defined by considering five values of the contours at equally spaced values between 0 and the cutoff, which is 1 here, drawn by linearly interpolating between $\bar{M}^{G}$ calculated at 20 values each for $G=$ $E$ and $I$. In each cluster, the smaller values are closer to the center. (b) Contours for values less than 0.1 are drawn for $\tau \bar{L}_{\mathrm{IC}}$. (c) Contours for values less than 0.04 are drawn for $\tau \bar{L}_{\mathrm{IC}}$. (d) Contours for values less than 0.1 are drawn for $\tau \bar{L}_{\mathrm{IC}^{\prime}}$.

FIG. 2. (a) Contours for values less than 0.04 are drawn for $\tau \bar{L}_{\mathrm{EC}}$. (b) Contours for values less than 0.04 are drawn for $\tau \bar{L}_{\mathrm{EC}}$. A right brace " $\}$ " signifies enclosure of other nested closed contours above and to the left of this brace.

FIG. 3. (a) Contours for values less than 0.04 are drawn for $\tau \bar{L}_{\mathrm{BC}}$. (b) Contours for values less than 0.04 are drawn for $\tau \overline{\underline{B}}_{\mathrm{BC}^{\prime}}$.

FIG. 4. (a) Contours for values less than 0.02 are drawn for $\tau \bar{L}_{F B C^{\prime}}$. (b) Contours for values less than 0.04 are drawn for $\tau \bar{L}_{\mathrm{BC}}$, but where $\bar{M}^{G}$ terms in the denominators of $F^{G}$ have been left out.

FIG. 5. Contours for values less than 0.04 are drawn for values of parameters in $\tau \overline{\underline{L}}_{\mathrm{BC}}$, but where $N=220$. Note that the $\bar{M}^{G}$ axes are accordingly stretched by a factor of 2 relative to the previous figures.

FIG. 6. (a) Contours for values less than 1.0 are drawn for the coefficients of $\left(\rho \nabla M^{E}\right)^{2}$ in $\tau L_{\mathrm{BC}}$. (b) Contours for values less than 1.0 are drawn for the coefficients of $\left(\rho \nabla M^{I}\right)^{2}$ in $\tau \underline{B}_{\mathrm{BC}}$. 


\begin{tabular}{|c|c|c|}
\hline$\overline{\overline{\left(\bar{M}^{E}, \bar{M}^{I}\right)}}$ & $\tau \underline{\underline{L}}_{\mathrm{BC}^{\prime}}$ & $\left\|\tau \bar{L}_{\mathrm{BC}^{\prime}, G G^{\prime}}\right\|$ \\
\hline$(-80,-30)$ & $7.23 \times 10^{-5}$ & 0.138 \\
\hline$(-64,-24)$ & 2.87 & $-5.95 \times 10^{-4}$ \\
\hline$(-48,-18)$ & 1.18 & $-5.22 \times 10^{-5}$ \\
\hline$(-32,-12)$ & 0.386 & $-4.53 \times 10^{-6}$ \\
\hline$(-16,-6)$ & 0.0760 & $-1.30 \times 10^{-7}$ \\
\hline$(0,0)$ & 0.0 & $1.07 \times 10^{-7}$ \\
\hline$(16,6)$ & 0.0520 & $-2.28 \times 10^{-7}$ \\
\hline$(32,12)$ & 0.162 & $-1.30 \times 10^{-6}$ \\
\hline$(48,18)$ & 0.233 & $-3.54 \times 10^{-6}$ \\
\hline$(64,24)$ & 0.146 & $-5.08 \times 10^{-6}$ \\
\hline$(80,30)$ & $7.71 \times 10^{-3}$ & $2.93 \times 10^{-5}$ \\
\hline
\end{tabular}

Table I. Lester Ingber 